\title{
Tonic stimulation of the pharyngeal mucosa causes pain and a reversible increase of inflammatory mediators
}

\author{
Bertold Renner • Gabi Ahne • Elke Grosan • \\ Birgit Kettenmann • Gerd Kobal • \\ Adrian Shephard
}

Received: 12 March 2013/Accepted: 26 August 2013/Published online: 15 September 2013

(C) The Author(s) 2013. This article is published with open access at Springerlink.com

\begin{abstract}
Objective and design To develop a model of the inflammatory component of non-infectious sore throat using tonic stimulation and quantification of inflammatory mediators in pharyngeal lavage fluid.

Material or subjects Forty-five healthy volunteers.

Treatment Cold dry air.

Method Tonic stimulation of the pharynx was achieved using a constant stream of cold dry air to the back of the throat. Following optimization of stimulation conditions (phase 1), pharyngeal pain, irritation, and swallowing discomfort were assessed using visual analog scales, and the concentration of inflammatory markers were measured in pharyngeal lavage fluid (phase 2).

Results Optimum conditions for tonic pharyngeal stimulation were cold dry air at $12{ }^{\circ} \mathrm{C}$, relative humidity $20 \%$, at a flow rate of $12 \mathrm{~L} / \mathrm{min}$ for $15 \mathrm{~min}$. Analysis of pharyngeal
\end{abstract}

Responsible Editor: Michael J. Parnham.

B. Renner and G. Ahne contributed equally to this work.

Electronic supplementary material The online version of this article (doi:10.1007/s00011-013-0663-7) contains supplementary material, which is available to authorized users.

B. Renner $(\bowtie) \cdot$ G. Ahne $\cdot$ E. Grosan · B. Kettenmann

G. Kobal

Department of Experimental and Clinical Pharmacology,

University of Erlangen-Nuremberg, Krankenhausstr. 9,

91054 Erlangen, Germany

e-mail: renner@pharmakologie.uni-erlangen.de

G. Kobal

Altria Client Services Inc., Richmond, VA, USA

A. Shephard

Reckitt Benckiser Healthcare Ltd, Slough, UK lavage fluid collected 5 min after stimulation showed significant increases in prostaglandin $\mathrm{E}_{2} \quad(P=0.018)$, thromboxane $\mathrm{B}_{2}(P<0.001)$, and substance $\mathrm{P}(P<0.001)$, but no increase in peptidoleukotriene. When the stimulus was removed, the level of inflammatory markers in pharyngeal lavage fluid returned to baseline by $30 \mathrm{~min}$ poststimulation. These objective measures mirrored subjective pain ratings.

Conclusions Tonic stimulation of the pharyngeal mucosa with cold dry air causes pain and an increase of inflammatory mediators which are reversible.

Keywords Cold dry air - Experimental model . Inflammation · Sore throat · Pharyngitis

\section{Introduction}

Pharyngitis (sore throat) can be caused by infectious agents (viruses, bacteria, and fungi) or physico-chemical (such as smoking, snoring, shouting, tracheal intubation, medications, or concomitant illness) or environmental (including indoor and outdoor air pollutants, temperature and humidity, and hazardous or occupational irritants) factors [1]. However, the underlying pathophysiology of noninfectious sore throat is not well understood, and experimental models with defined conditions and objective endpoints are needed to study the mechanisms and evaluate treatment strategies [1].

Many of the currently available models are not specific for sore throat or use subjective endpoints. There are several models for the study of non-allergic rhinitis, but they mainly induce nasal symptoms-examples include intranasal challenge with histamine [2], capsaicin [3], or methacholine [4], as reviewed previously [5]. While nasal 
provocation with bradykinin induces sore throat, it also induces rhinitis [6]. The rating of sore throat pain $[7,8]$ is specific for sore throat, but the endpoint is subjective. In preclinical settings, there is no animal model available to test drugs for the treatment of sore throat.

Nasal stimulation with cold dry air is an established model for rhinitis [5], and may be a suitable model for the inflammatory component of some etiologies of non-infectious sore throat when applied to the pharynx. When introduced to the nasal cavity of volunteers, a stream of air induces pain, the intensity of which varies depending on humidity and temperature [9], and potentially air flow rate. Previous work in our laboratory used cold dry air $\left(22{ }^{\circ} \mathrm{C}\right.$, $20 \%$ relative humidity, $8 \mathrm{~L} / \mathrm{min}$ ) to experimentally induce rhinitis in healthy volunteers [10]. The assessment of inflammatory mediators in nasal lavage fluid provides an objective measure of inflammation [11] and quantifies the response to nasal cold dry air provocation [10]. Pain induced by nasal application of cold dry air has been used to assess the anti-inflammatory and analgesic properties of acetaminophen, ibuprofen, ketoprofen, and azapropazone [12-15].

Pharyngeal lavage has been used to investigate mucosal inflammation in the pharynx of patients with sleep-related disordered breathing [16]. In the current study, we developed a model of pharyngeal inflammation using local application of cold dry air, with quantification of inflammatory mediators in pharyngeal lavage fluid.

\section{Materials and methods}

The study was approved by the University of ErlangenNuremberg's institutional review board, and was conducted in agreement with the Declaration of Helsinki (Tokyo amendment). Parts of the data are published in a thesis [17].

\section{Subjects}

A total of 45 healthy volunteers participated, with a mean age of $25.2 \pm 1.9$ years (range 22-34 years), including 23 males and 22 females. Five subjects participated in preliminary experiments. Subsequently, 20 subjects participated in phase 1 and 20 subjects participated in phase 2. They were recruited at the Department of Experimental and Clinical Pharmacology and Toxicology, University of Erlangen-Nuremberg, Germany. The study was conducted between October 1998 and June 2000, and prior to commencement, written informed consent was obtained from all subjects. The participants received 100 DM (about 50 Euro) for each experimental session.

Inclusion criteria included volunteers who were physically and mentally healthy, aged $18-45$ years, and of normal body weight (Broca Index $\pm 25 \%$ ). Excluded were those subjects with evidence or suspicion of any clinical abnormality, any acute or chronic infection or allergy requiring therapy, those who were taking concomitant medication (except contraceptives) or who had taken medication within the previous 4 weeks, and those with relevant loss of blood within the last month. Smokers (more than 15 cigarettes/day) were excluded, as were people dependant on drugs or ingesting more than $60 \mathrm{~g}$ alcohol/day, and those with any diet (including vegetarian) or lifestyle that would interfere with the study. Also excluded were people with known or suspected non-compliance, pregnant or lactating women, and those with actual or anamnestic bronchial asthma.

All subjects underwent pre-study screening to confirm their health, including a general medical examination, measurement of vital signs, and 12-lead electrocardiogram.

In a training session prior to the study, the subjects were familiarized with the experimental procedures and with velopharyngeal closure, a breathing technique that avoids respiratory flow within the nasal cavity during stimulation [18]. This ensures the delivery of the stimulus is independent of breathing through the nose which could otherwise influence its intensity and hence the subject's response.

\section{Tonic stimulation of the pharynx}

Stimulation of the pharynx was achieved using a constant stream of cold dry air to the back of the throat (Fig. 1). The optimum conditions for this were established in phase 1 . Low relative humidity ( $20 \%$ ) was achieved by passing the airstream through a bottle filled with silica gel. For thermostabilization, the bottle and tubing were located in a thermostat. All materials were made of glass or Teflon ${ }^{\circledR}$.

During all studies, the subjects were comfortably seated in an air-conditioned room with white noise used to provide acoustic shielding. Subjects fasted for at least $6 \mathrm{~h}$

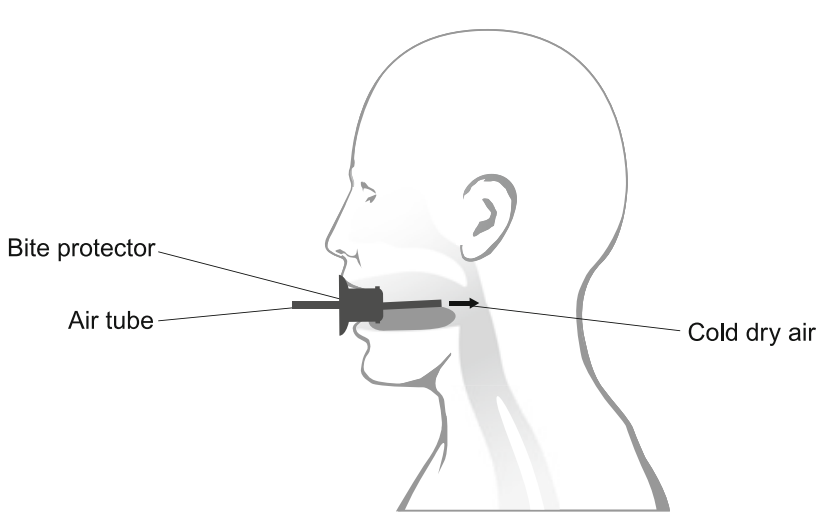

Fig. 1 Schematic diagram showing the apparatus used to apply cold dry air to the pharynx of volunteers 
before each experiment. They brushed their teeth $2 \mathrm{~h}$ before each experiment with the standard toothbrush and toothpaste provided. Thereafter, no smoking and only water was allowed. Subjects cleaned their mouth with water $15 \mathrm{~min}$ before each session.

In order to stabilize vigilance during the experiments, the subjects were required to perform a tracking task on a video screen [19]. Using a joystick, they had to keep a small square within a randomly moving larger square. By measuring this 'tracking performance' it was possible to monitor the vigilance of the subjects.

Phase 1: optimization of tonic pharyngeal stimulation

A range of conditions for stimulation of the pharynx were investigated in pilot experiments in five subjects. Optimum conditions for stimulation of the pharynx were identified in phase 1 of the study in 20 subjects: at a relative humidity of $20 \%$, different combinations of air flow, temperature, and duration of stimulus were investigated (Table 1). Pain intensity and aversion were assessed by a visual analog scale (VAS) throughout stimulation. Each subject underwent 15 sessions (randomized, single blind) in total (Table 1), with one session per day and at least 2 days between each session.

Phase 2: effect of tonic pharyngeal stimulation on inflammatory markers and pain

In phase 2 , the effect of the optimum cold dry air conditions determined in phase $1\left(12{ }^{\circ} \mathrm{C}\right.$, relative humidity

Table 1 The conditions which were investigated during phase 1 to optimize pharyngeal stimulation, at $20 \%$ relative humidity

\begin{tabular}{llll}
\hline Condition & $\begin{array}{l}\text { Air flow } \\
(\mathrm{L} / \mathrm{min})\end{array}$ & $\begin{array}{l}\text { Temperature } \\
\left({ }^{\circ} \mathrm{C}\right)\end{array}$ & $\begin{array}{l}\text { Duration of } \\
\text { stimulus }(\mathrm{min})\end{array}$ \\
\hline 1 & 12 & 12 & 15 \\
2 & 12 & 12 & 5 \\
3 & 12 & 12 & 10 \\
4 & 12 & 12 & 20 \\
5 & 12 & 12 & 25 \\
6 & 6 & 12 & 15 \\
7 & 8 & 12 & 15 \\
8 & 10 & 12 & 15 \\
9 & 14 & 12 & 15 \\
10 & 12 & 4 & 15 \\
11 & 12 & 6 & 15 \\
12 & 12 & 8 & 15 \\
13 & 12 & 10 & 15 \\
14 & 12 & 15 & 15 \\
15 & 12 & 18 & 15 \\
\hline
\end{tabular}

$20 \%$, at $12 \mathrm{~L} / \mathrm{min}$ for $15 \mathrm{~min}$ ) on pharyngeal pain, irritation, and swallowing discomfort (VAS) and concentration of inflammatory markers in pharyngeal lavage fluid was assessed in 20 subjects. Each subject underwent one session of tonic pharyngeal stimulation on each of 2 days.

Assessment of subject's perception of pain and discomfort

During stimulation, every $60 \mathrm{~s}$ during the application of the air flow to the pharynx, the subjects rated the intensity of and aversion to pain on a VAS displayed on a computer screen [19]. The pain intensity scale ran from 0 (no pain) to 100 (maximal imaginable pain) and the aversion scale ran from 0 (no aversion) to 100 (maximal aversion). The pain intensity scale was the primary indicator, whilst the aversion scale was used to check for non-pain adverse events or discomfort.

After stimulation, throat pain, throat irritation, and discomfort while swallowing were assessed every $10 \mathrm{~min}$ for 80-90 min post-stimulation. The VAS for these ran from 0 (no pain) to 100 (maximal imaginable pain), from 0 (no irritation) to 100 (maximal irritation), and from 0 (no swallowing discomfort) to 100 (maximal swallowing discomfort).

Pharyngeal lavage technique

Pharyngeal lavage was performed immediately before (at $0 \mathrm{~min}$ ), and at 5 and $30 \mathrm{~min}$ after pharyngeal stimulation. The subjects were asked to gargle with $10 \mathrm{~mL}$ of warmed $\left(37^{\circ} \mathrm{C}\right)$ Ringer's solution (pH 5-7) for $10 \mathrm{~s}$. After collecting the lavage fluid it was immediately centrifuged (10 min, $1,700 \mathrm{rpm}, 4{ }^{\circ} \mathrm{C}$ ) to separate the cell pellet, then frozen at $-80^{\circ} \mathrm{C}$ until further analysis.

\section{Measurement of inflammatory mediators}

Pilot experiments in five subjects determined if inflammatory mediators could be measured in pharyngeal lavage fluid. The concentrations of inflammatory mediators [prostaglandin $\mathrm{E}_{2} \quad\left(\mathrm{PGE}_{2}\right)$, peptidoleukotriene (PLT), thromboxane $\mathrm{B}_{2}$ (TXB), and substance $\mathrm{P}$ (SP)] in pharyngeal lavage fluid were measured by enzyme-linked immunosorbent assay (ELISA) (Cayman Chemical Company, Ann Arbor, MI, USA). These mediators were selected following their responsiveness to nasal stimulation with cold dry air [10], and the presence of substance P in sensory nerve fibres of the upper respiratory tract [20]. After defrosting, analysis of TXB and SP was conducted using undiluted samples, whilst those for $\mathrm{PGE}_{2}$ and PLT were diluted 1:10 with Ringer Lactate solution (Braun, Melsungen, Germany). The intensity of colour change was 
determined spectrophotometrically (microplate reader MR2100, Firma Dynex Technologies, Düsseldorf, Germany). Results outside the validated range were excluded and treated as missing data.

\section{Statistical analyses}

Outcome measures were subjective ratings of pain intensity and aversion during stimulation, subjective ratings of pain, irritation, and swallowing discomfort after stimulation, and pre- and post-stimulation concentrations of inflammatory mediators ( $\mathrm{PGE}_{2}, \mathrm{PLT}, \mathrm{TXB}$, and $\left.\mathrm{SP}\right)$ in lavage fluid.

All statistical analyses were conducted using IBM SPSS Statistics 21 (Chicago, IL, USA). Descriptive analyses included calculation of mean, range, standard deviation, and standard error. Repeated measures ANOVA was used for inflammatory mediators in order to detect stimulation and recovery effects over time. Student's $t$ tests were used as post hoc tests, adjusting each $P$ value according to Bonferroni. Spearman rank correlation coefficient was used for analysis of correlations between increases of different mediators and pain ratings after cold dry air stimulation compared with baseline. Data are presented as means with $95 \%$ confidence intervals $(\mathrm{CI}) . P<0.05$ was considered significant.

\section{Results}

Phase 1: optimization of tonic pharyngeal stimulation

When combinations of stimulation parameters (Table 1) were investigated in phase $1(n=20)$ it was clear that the greatest intensity of pharyngeal pain was achieved using $12 \mathrm{~L} / \mathrm{min}$, at $12{ }^{\circ} \mathrm{C}$, for $15 \mathrm{~min}$ (condition 1 in Table 1 ; Fig. 2a). Warming the air to $18{ }^{\circ} \mathrm{C}$ considerably reduced the pain intensity (condition 15 in Table 1; Fig. 2a). Cooling the airflow to below $12{ }^{\circ} \mathrm{C}$ (conditions 10-13) or increasing the duration of the stimulus (conditions 4-5) did not further increase the pain intensity (Fig. 2a). Ratings of aversion throughout generally followed the same trends (Fig. 2b). The optimum conditions for tonic pharyngeal stimulation were therefore determined to be $12 \mathrm{~L} / \mathrm{min}$ at $12{ }^{\circ} \mathrm{C}$ for $15 \mathrm{~min}$.

Phase 2: effect of tonic pharyngeal stimulation on inflammatory markers and pain

In phase $2(n=20)$, there was a significant change in all inflammatory mediators over time $\left(\mathrm{PGE}_{2}: d f=2, F=5.7\right.$, $P=0.005$; TXB: $d f=2, F=8.5, P<0.001 ; \mathrm{SP}: d f=2$, $F=18.5, P<0.001$, PLT: $d f=2, F=4.4, P=0.028)$. Post hoc analysis of pharyngeal lavage fluid collected 5 min after tonic pharyngeal stimulation showed significant
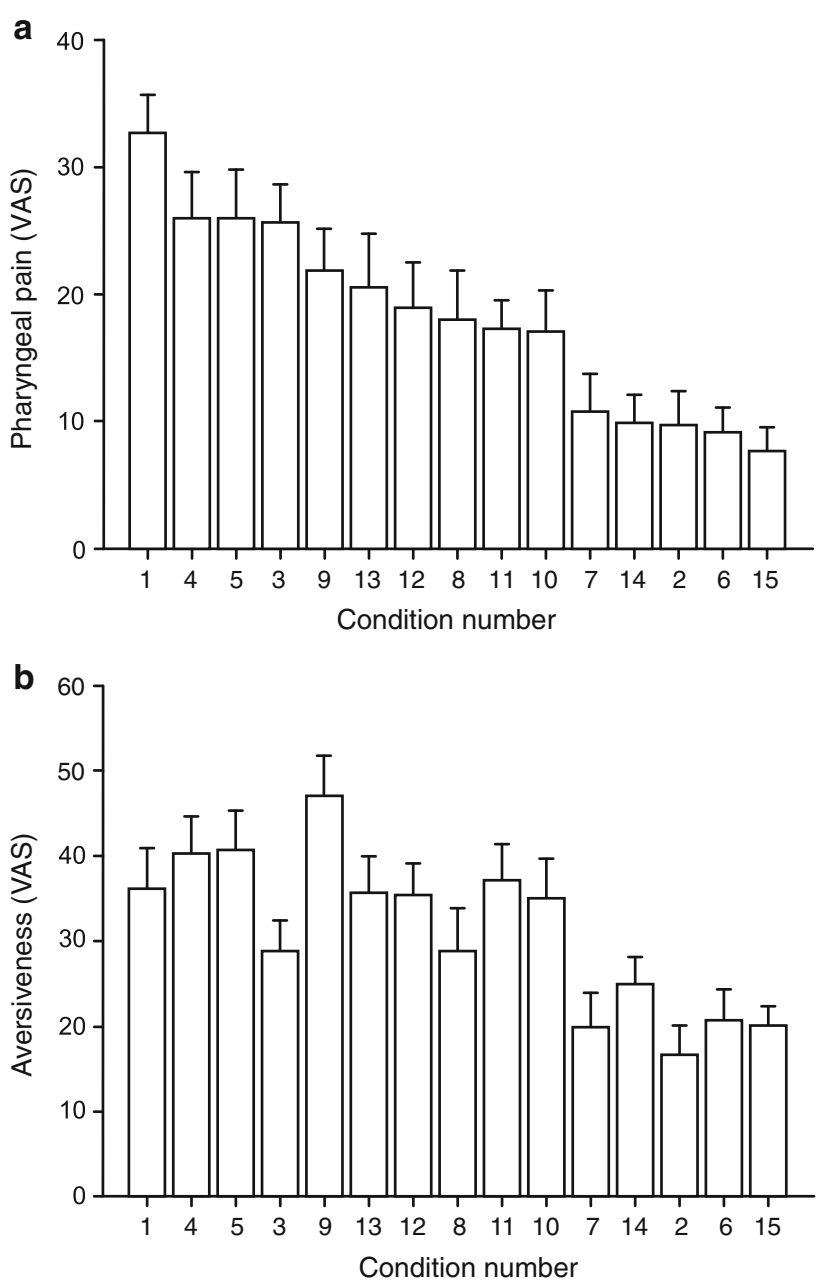

Fig. 2 Pharyngeal pain intensity (a) and aversion (b) (mean $95 \%$ confidence interval), measured on visual analog scales (VAS), during phase 1 tonic pharyngeal stimulation with cold dry air varying in flow rate, temperature, and duration of stimulus. For details of the air flow characteristics for each of the 15 experiments see Table 1

increases in $\mathrm{PGE}_{2}(P=0.018)$, TXB $(P<0.001)$, and SP $(P<0.001)$ compared with baseline (Fig. 3$)$. When the stimulus was removed, the level of inflammatory markers in pharyngeal lavage fluid returned to baseline by $30 \mathrm{~min}$ post-stimulation (Fig. 3). There was no significant increase in PLT upon tonic pharyngeal stimulation (change from baseline $161 \mathrm{pg} / \mathrm{mL}$ (95\% CI 52-270 pg/mL) and $105 \mathrm{pg} /$ $\mathrm{mL}(95 \%$ CI $10-200 \mathrm{pg} / \mathrm{mL})$ for first and second time point after stimulation, $n=14$ ).

The phase 2 VAS results $(n=20)$ show the pharyngeal pain (Fig. 4a) and stimulus aversion (Fig. 4d, supplement) during tonic pharyngeal stimulation $\left(12 \mathrm{~L} / \mathrm{min}\right.$ at $12{ }^{\circ} \mathrm{C}$ for $15 \mathrm{~min}$ ), which increases progressively from the onset of the stimulus and throughout its duration. After removal of the stimulus, irritation (Fig. 4b) and swallowing difficulty (Fig. 4c) peak and then progressively improve over the next 80-90 min, and pain reduces to baseline (Fig. 4a). 



Fig. 3 Change (mean $95 \%$ confidence interval, $n=20$ ) in a prostaglandin E2, b thromboxane B2, c substance $\mathrm{P}$ in pharyngeal lavage fluid of healthy volunteers after tonic pharyngeal stimulation (the grey shaded area) with cold dry air $\left(12{ }^{\circ} \mathrm{C}, 20 \%\right.$ humidity at $12 \mathrm{~L} / \mathrm{min}$ for $15 \mathrm{~min}$ ). Results are the means of two tests. Student's $t$ test was used with Bonferroni-adjusted $P$ values shown
Correlation analysis showed that the increased pain ratings during cold dry air stimulation and decreased ratings thereafter coincided with changes in mediator release. This was significant for TXB $(P=0.042 ; r=0.231)$ and $\mathrm{SP}(P=0.034 ; r=0.237)$, but was not significant for PLT and $\mathrm{PGE}_{2}$.

\section{Discussion}

This study shows that tonic stimulation of the pharyngeal mucosa with cold dry air causes pain, irritation, and discomfort whilst swallowing and an increase of inflammatory mediators, which is reversible. This is the first sore throat model that is both specific to the pharynx and includes an objective endpoint (pharyngeal inflammatory markers). Other currently available techniques $[7,8]$ do not routinely measure inflammatory mediators and rely on assessment of the physical appearance of inflammation.

The properties of an ideal pain model have previously been described [21]. An ideal stimulus should exhibit minimal neurohistologic variation between individuals, should be measureable, closely associated with the changes which produce inflammation, provoke minimal tissue damage, show a relation to pain intensity, provide information about discrimination between stimuli, result in repeatable stimulation without temporal interaction, be applied easily, allow a quantifiable determination of the quality of inflammation, be sensitive to agents of low analgesic power, show dose-related effects of anti-inflammatory drugs, and be applicable for both man and animal [10]. The current sore throat pain model satisfies many of these criteria, although its sensitivity and dose-response to anti-inflammatory agents was not tested.

The optimum conditions for pharyngeal stimulation were confirmed to be cold dry air at $12 \mathrm{~L} / \mathrm{min}, 20 \%$ relative humidity and $12{ }^{\circ} \mathrm{C}$, with a 15 -min stimulus duration. These conditions maximized the subjective pharyngeal pain reported by the volunteers, and hence maximized the sensitivity of the model. The conditions required for optimum tonic stimulation of the pharynx are somewhat harsher than those employed for the nasal cavity $\left(22{ }^{\circ} \mathrm{C}, 20 \%\right.$ relative humidity, $8 \mathrm{~L} / \mathrm{min}$ ) [10], and this may be due to the relative sensitivity of the mucosa at these locations. There may be differences in innervation and/or neuropeptide release [20], and the clearance of inflammatory mediators and neuropeptides on the pharyngeal mucosa may also be enhanced by saliva production, which is not the case in the nasal cavity. The single-layer respiratory epithelium (pseudostratified) changes to a multiple-layer epithelium in the lower pharynx, and this could also influence sensitivity.

The increase in inflammatory markers in pharyngeal lavage fluid upon tonic stimulation with cold dry air 

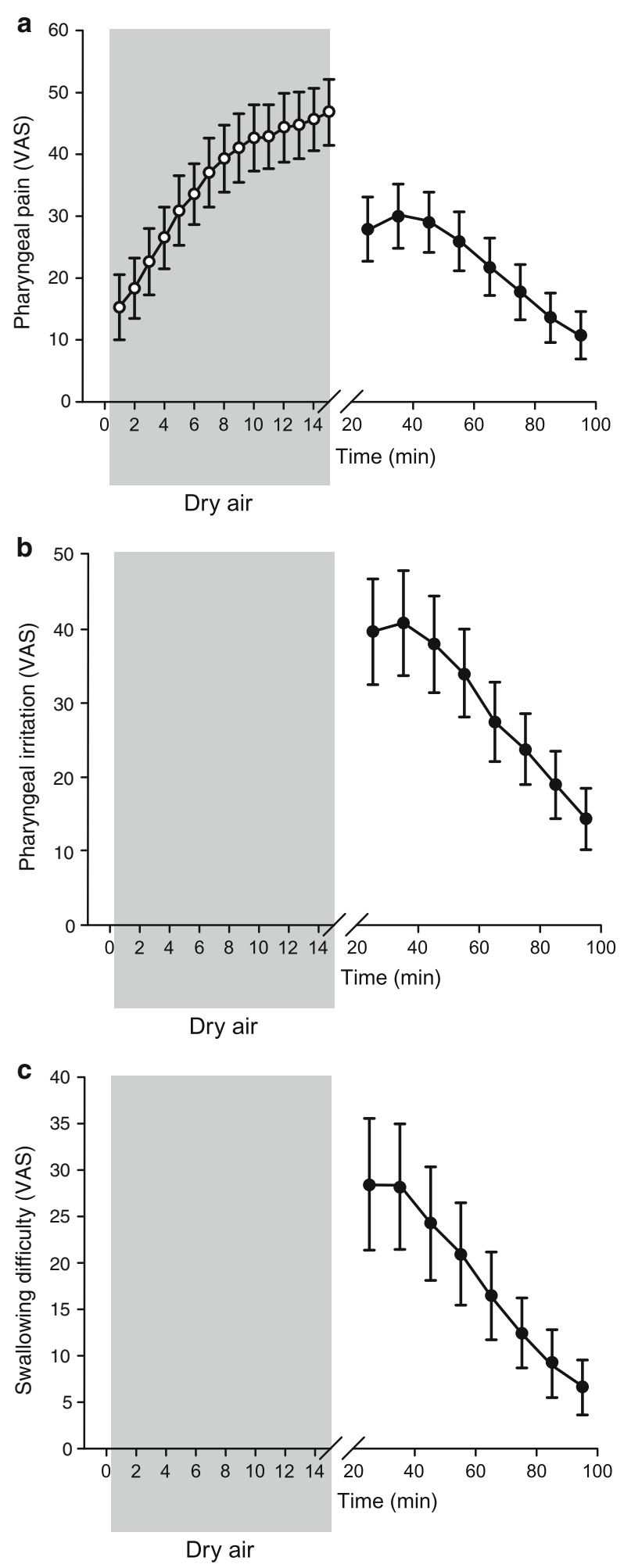

Fig. 4 Change (mean $95 \%$ confidence interval, $n=20$ ) in pharyngeal pain intensity (a), irritation (b) and swallowing difficulty (c) of healthy volunteers, measured on visual analog scale (VAS), during (the grey shaded area) and after tonic pharyngeal stimulation with cold dry air $\left(12{ }^{\circ} \mathrm{C}, 20 \%\right.$ humidity at $12 \mathrm{~L} / \mathrm{min}$ for $\left.15 \mathrm{~min}\right)$. Results are the means of two tests. VAS for pharyngeal irritation and swallowing difficulty were not recorded during the stimulation period provides an objective measure that mirrors the subjective pain ratings. Both pain and inflammatory markers increased upon stimulation then returned to baseline shortly afterwards. The timing of the peak of inflammatory markers coincided with the pain measures. However, inflammatory mediators returned to baseline more quickly (at about 30 min after the stimulus was removed) compared with pain measures (at about 80-90 min after the stimulus was removed). In this regard, we could not exclude any dilution effects caused by repetitive lavage sampling, which will be pronounced during post-stimulation periods. Our analysis reflected this time shift as only $\mathrm{SP}$ and TXB but not $\mathrm{PGE}_{2}$ or PLT release correlated with changes in pain ratings. However, a moderate correlation is typical for subjective ratings and was also observed in a previous study, following stimulation of the nasal mucosa with cold dry air [22].

In the current study, $\mathrm{PGE}_{2}, \mathrm{TXB}$, and SP levels in pharyngeal lavage fluid were significantly increased by cold dry air; but there was no significant effect on PLT. In contrast, a previous study found significant increases in PLT in nasal lavage fluid when cold dry air was applied to the nasal cavity, although increases in $\mathrm{PGE}_{2}$ and TXB failed to reach significance [10]. The differences between studies may be due to variability in the data or missing values. The data are currently insufficient to determine if the differences in mediator responses (within the current study, and between studies) are due to anatomical location, stimulus characteristics, or other methodological variation. Previous data on pharyngeal lavage are not informative as they are limited to inflammatory cell counts rather than inflammatory mediators [16]. The significant increases in pharyngeal $\mathrm{PGE}_{2}, \mathrm{TXB}$, and $\mathrm{SP}$ in the current study show that these mediators are implicated in the inflammation induced by cold dry air. Whereas SP is known to be involved in nociception and the development of hyperalgesia, $\mathrm{PGE}_{2}$ and TXB may have contrary physiological effects (for example, bronchial relaxation versus constriction). We included these mediators because $\mathrm{PGE}_{2}$ and TXB may be used to quantify the effect of selective and unselective cyclooxygenase inhibitors [23]. As we measured the acute release of these mediators (during the 15-min stimulation period), there is limited information about lipid mediator production which takes 30 min or longer. However, since we observed rapid recovery of pain and mediator release, the stimulation could potentially be repeated several times per day in order to obtain longerterm effects on inflammatory mediator induction or regulation. Other lipid mediators are likely to be involved but were not analyzed in the current study. Regulators of pharyngeal inflammation (pro-inflammatory and antiinflammatory) warrant further study, and the current model may provide a tool for this. From human studies of nasal challenge with cold dry air there is a clear suggestion of 
generation of leukotrienes and kinins; although only in predisposed subjects with rhinitis [22, 24].

The lack of PLT response in the current study may be due to a higher degradation of PLT or greater differences in local production/secretion of PLT. The ELISA kit used for analysis of PLT in the current study detected leukotriene (LT) $\mathrm{C}_{4}, \mathrm{LTD}_{4}$ and the degraded metabolite $\mathrm{LTE}_{4}$. Since the kit provides a high specificity for $\mathrm{LTC}_{4}$ and $\mathrm{LTD}_{4}(100 \%)$ and a lower specificity for $\mathrm{LTE}_{4}$ (below $70 \%$ ), differences in degradation state may contribute to the PLT data variability observed in the current study. The degradation of $\mathrm{LTE}_{4}$ to undetectable metabolites is incomplete in bronchoalveolar samples, but the degradation time response is currently unknown in samples containing saliva. Methodological differences between the current study and the nasal lavage study [10] include different sample volumes (10 vs. $6 \mathrm{~mL}$, respectively), which could affect sensitivity.

In summary, the cold dry air model presented here provides a well-controlled, easily-applied technique for inducing reversible sore throat pain that is specific to the pharynx and can be measured objectively. This model will be of benefit for the future development of analgesics for alleviating non-infectious sore throat of some etiologies with an inflammatory component, the investigation of environmental causes of sore throat [1] including allergic and nonallergic states in environmental medicine, as well as toxicology exposure studies with defined stimulus conditions in addition to cold dry air.

Acknowledgments Editorial assistance was provided by Elements Communications Ltd, supported by Reckitt Benckiser Healthcare International Ltd.

Open Access This article is distributed under the terms of the Creative Commons Attribution License which permits any use, distribution, and reproduction in any medium, provided the original author(s) and the source are credited.

\section{References}

1. Renner B, Mueller CA, Shephard A. Environmental and noninfectious factors in the aetiology of pharyngitis (sore throat). Inflamm Res. 2012;61:1041-52.

2. Bedard PM, Jobin M, Clement L, Mourad W, Hebert J. Evaluation of nonspecific nasal reactivity to histamine during and after natural ragweed pollen exposure. Am J Rhinol. 1989;3:211-5.

3. Bascom R, Kagey-Sobotka A, Proud D. Effect of intranasal capsaicin on symptoms and mediator release. J Pharmacol Exp Ther. 1991;259:1323-7.

4. Borum P. Nasal methacholine challenge. A test for the measurement of nasal reactivity. J Allergy Clin Immunol. 1979;63: 253-7.

5. Philip G, Togias AG. Nonallergic rhinitis. Pathophysiology and models for study. Eur Arch Otorhinolaryngol. 1995;252(Suppl 1):S27-32.

6. Proud D, Reynolds CJ, Lacapra S, Kagey-Sobotka A, Lichtenstein LM, Naclerio RM. Nasal provocation with bradykinin induces symptoms of rhinitis and a sore throat. Am Rev Respir Dis. 1988;137:613-6.

7. Schachtel BP, Fillingim JM, Thoden WR, Lane AC, Baybutt RI. Sore throat pain in the evaluation of mild analgesics. Clin Pharmacol Ther. 1988;44:704-11.

8. Schachtel BP, Pan S, Kohles JD, Sanner KM, Schachtel EP, Bey M. Utility and sensitivity of the sore throat pain model: results of a randomized controlled trial on the COX-2 selective inhibitor valdecoxib. J Clin Pharmacol. 2007;47:860-70.

9. Lötsch J, Ahne G, Kunder J, Kobal G, Hummel T. Factors affecting pain intensity in a pain model based upon tonic intranasal stimulation in humans. Inflamm Res. 1998;47:446-50.

10. Mohammadian P, Schaefer D, Hummel T, Kobal G. Experimentally induced nasal irritation. Rhinology. 1999;37:175-8.

11. Koren HS, Hatch GE, Graham DE. Nasal lavage as a tool in assessing acute inflammation in response to inhaled pollutants. Toxicology. 1990;60:15-25.

12. Hummel T, Huber H, Menzel S, Kobal G. Tonic versus phasic pain: dose-related effects of ketoprofen. Eur J Clin Pharmacol. 1995;49:7-14.

13. Kobal G, Hummel C, Gruber M, Geisslinger G, Hummel T. Dose-related effects of ibuprofen on pain-related potentials. Br J Clin Pharmacol. 1994;37:445-52.

14. Lötsch J, Mohammadian P, Hummel T, Florin S, Brune K, Geisslinger G, Kobal G. Effects of azapropazone on pain-related brain activity in human subjects. $\mathrm{Br} \mathrm{J}$ Clin Pharmacol. 1995;40:545-52.

15. Renner B, Clarke G, Grattan T, Beisel A, Mueller C, Werner U, Kobal G, Brune K. Caffeine accelerates absorption and enhances the analgesic effect of acetaminophen. J Clin Pharmacol. 2007;47:715-26.

16. Hauber HP, Rüller S, Müller E, Hansen E, Zabel P. Pharyngeal lavage lymphocytosis in patients with obstructive sleep apnea: a preliminary observation. PLoS ONE. 2011;6:e16277.

17. Grosan E. Erzeugung eines Entzündungsschmerzes durch tonische Reizung der Rachenschleimhaut gesunder Probanden mit kalter, trockener Luft (Inducing inflammatory pain by tonic pharyngeal stimulation with cold dry air in healthy subjects). Thesis, Erlangen Germany: Friedrich-Alexander University Erlangen-Nuremberg; 2005.

18. Kobal G, Hummel T. Olfactory evoked potentials in humans. In: Getchell TV, Doty RL, Bartoshuk LM, Snow JB, editors. Smell and taste in health and disease. New York: Raven Press; 1991.

19. Kobal G, Hummel C, Nuernberg B, Brune K. Effects of pentazocine and acetylsalicylic acid on pain-rating, pain-related evoked potentials and vigilance in relationship to pharmacokinetic parameters. Agents Actions. 1990;29:342-59.

20. Hauser-Kronberger C, Hacker GW, Franz P, Albegger K, Dietze O. CGRP and substance $P$ in intraepithelial neuronal structures of the human upper respiratory system. Regul Pept. 1997;72:79-85.

21. Beecher HK. Measurement of subjective responses: quantitative effects of drugs. New York: Oxford University Press; 1959.

22. Togias AG, Naclerio RM, Proud D, Fish JE, Adkinson NF Jr, Kagey-Sobotka A, Norman PS, Lichtenstein LM. Nasal challenge with cold, dry air results in release of inflammatory mediators. Possible mast cell involvement. J Clin Invest. 1985;76:1375-81.

23. Patrignani P, Panara MR, Greco A, Fusco O, Natoli C, Lacobelli S, Cipollone F, Ganci A, Créminon C, Maclouf J, et al. Biochemical and pharmacological characterization of the cyclooxygenase activity of human blood prostaglandin endoperoxide synthases. J Pharmacol Exp Ther. 1994;271:1705-12.

24. Togias AG, Naclerio RM, Peters SP, Nimmagadda I, Proud D, Kagey-Sobotka A, Adkinson NF Jr, Norman PS, Lichtenstein LM. Local generation of sulfidopeptide leukotrienes upon nasal provocation with cold, dry air. Am Rev Respir Dis. 1986;133:1133-7. 